\title{
8 APRA
}

Agricultural Policy Research in Africa
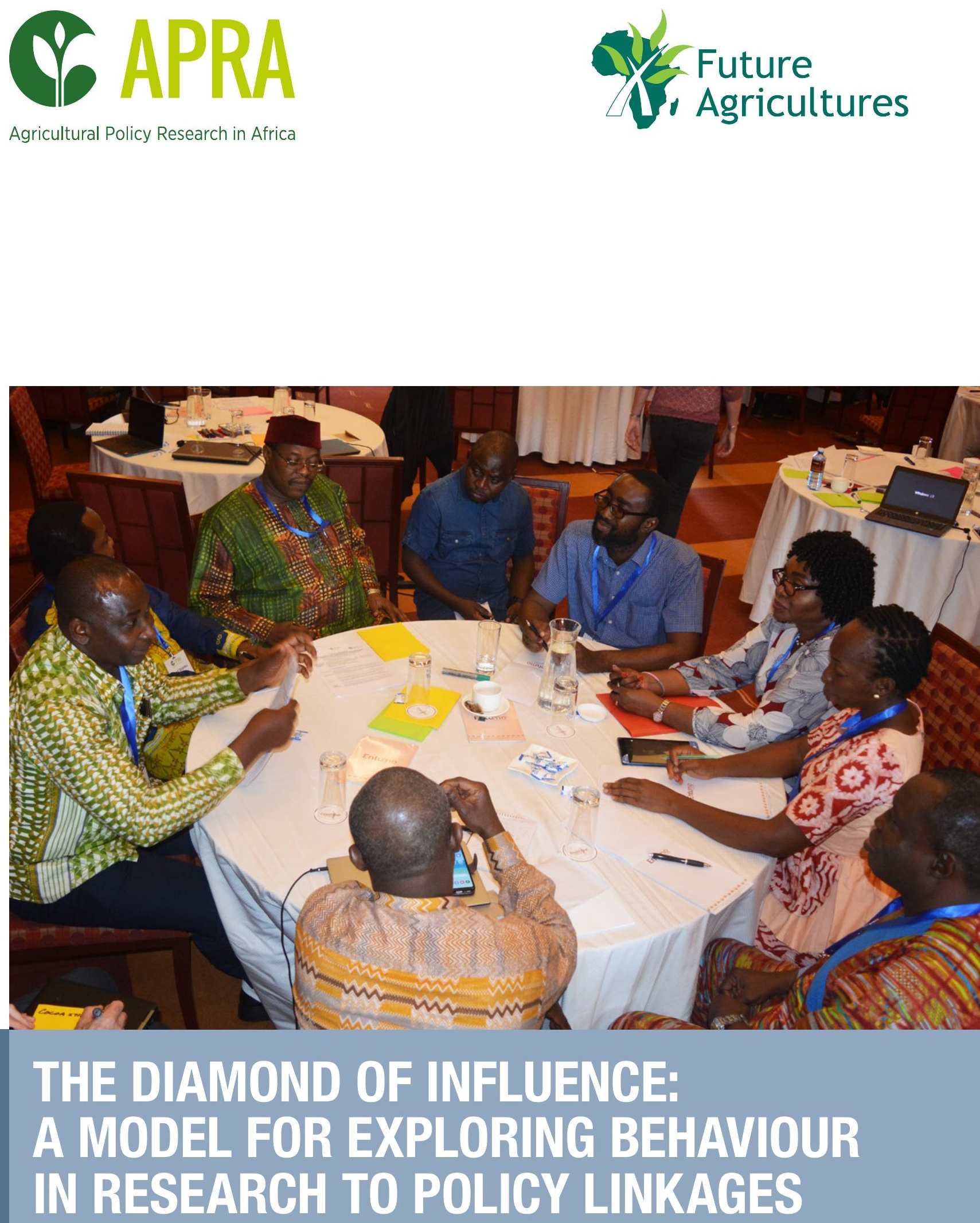

Louise Clark

ALRE Research Note 1

October 2020 


\section{Introduction}

This learning paper presents initial analysis of the emerging research to policy linkages within the Agricultural Policy Research in Africa (APRA) programme of the Future Agricultures Consortium, which is funded by the Foreign, Commonwealth \& Development Office (FCDO).

APRA has an innovative monitoring, evaluation and learning approach known as the 'Accompanied Learning on Relevance and Effectiveness' (ALRE), which is being delivered by a small team of embedded evaluation specialists. This paper discusses how ALRE has applied the COM-B (Capability, Opportunity, Motivation and Behaviour) (Mayne 2018; Mayne 2016; Michie, van Stralen and West 2011) model of behaviour change to explore the interactions and influencing strategies between researchers and policymakers in the context of agricultural policy research in Africa. These insights have produced the Diamond of Influence, a new ALRE-adapted model, which applies each of the COM-B elements to discuss the different aspects of research to policy processes, drawing on examples of how researchers in each of the APRA focus countries (Ethiopia, Ghana, Malawi, Nigeria, Tanzania and Zimbabwe) are engaging in policy spaces. These examples emphasise stakeholder engagement as key to ensuring the relevance, effectiveness and sustainability of APRA research.

The aim of this paper is to support critical thinking and to generate practical insights in order to help increase the relevance of APRA evidence in the context of local, national and regional policy priorities. The paper also reflects on the most effective mechanisms and key messages to identify, reach and influence decisionmakers. This discussion of different engagement strategies seeks to generate insights for researchers as they respond to evolving policy contexts and provide a framework for analysing APRA's contribution to political discourse and decisions on pro-poor agricultural commercialisation.

\section{Box 1: DAC definitions of relevance and effectiveness}

Relevance - the extent to which the intervention objectives and design respond to beneficiaries', global, country, and partner/institution needs, policies, and priorities, and continue to do so if circumstances change.

Effectiveness - the extent to which the intervention achieved, or is expected to achieve, its objectives, and its results, including any differential results across groups.
ALRE: A mechanism to increase research relevance and effectiveness

ALRE is a developmental evaluation approach (Patton 2010), which aims to deliver accountability and learning by finding the right balance between the objectivity and rigour of external evaluation with the strong programmatic knowledge and working relationships of embedded monitoring, evaluation and learning (MEL) staff. ALRE has been designed to support the APRA research partnership to deliver independence, rigour and accountability with ongoing reflection on programme performance and advice to improve pathways to impact. ALRE's objectives are to:

- increase APRA's relevance, effectiveness and overall outcomes by providing an informed, independent and iterative 'critical friend' function;

- conduct robust outcome tracing to improve the rigour and quality of outcome reporting; and

- generate learning on research-evidence-policy linkages to inform future programme design.

This paper aims to provide a working definition of what relevance and effectiveness mean in the context of APRA research, and to provide a model - the Diamond of Influence - to assess progress and performance. Practical examples emerging from across APRA focal countries, discussed with reference to this analytical model, are used to reflect upon APRA teams' research and engagement strategies and consider how the different dimensions of the model relate to their experience. The evidence presented in this paper is based upon researcher presentations given at the APRA Annual Review and Planning Workshop held in Naivasha, Kenya, in December 2019, and which was validated with country teams during 2020.

The starting point for discussing relevance and effectiveness are the Development Assistance Committee (DAC) of the Organisation for Economic Co-operation and Development (OECD) (OECD 2019; 1991) evaluation criteria, which were revised in 2019. The definitions provided in Box 1 reflect the 2019 updated criteria.

These definitions provide a starting point, but need to be further developed to reflect the specific context of APRA's research and objectives. When considering relevance it is key to ask: Relevant to what? To whom? At what level? For effectiveness, the emphasis needs to be on the actual use of research evidence to influence policy, investment and behavioural change. Prioritising relevance and effectiveness is key to ensuring APRA's legacy, which has two aspects: firstly, the capacity, relationships and networks that APRA will leave behind or provide as a foundation for future initiatives; and, secondly, building awareness and ownership of APRA evidence to support the uptake and use of APRA research findings in policy processes beyond the end of the APRA programme. 
The following section describes how the COM-B model has been applied to explore the different dimensions of research to policy linkages. This paper focusses specifically on emerging insights to guarantee the relevance of APRA research to policy conversations and opportunities.

\section{COM-B as a framework for exploring research to policy linkages}

The COM-B model explores the dynamics of factors that interact and support behaviour change as a combination of 'Capabilities, Opportunities and Motivations'. The model was originally developed through Michie et al.'s (2011) work on the 'Behaviour Change Wheel', which explored the relationships between evidence-based practice and behaviour change in public health.

"Capability is defined as the individual's psychological and physical capacity to engage in the activity concerned. It includes having the necessary knowledge and skills. Motivation is defined as all those brain processes that energise and direct behaviour, not just goals and conscious decision-making. It includes habitual processes, emotional responding, as well as analytical decision-making. Opportunity is defined as all the factors that lie outside the individual that make the behaviour possible or prompt it." (Michie et al. 2011: 4)
Michie et al. (2011) go on to identify sub-divisions for each of these components - physical and psychological capabilities; physical and social opportunities; and reflective and automatic motivations - that support the targeting of interventions. For the purpose of this paper, we will work with the COM-B principles, expanding upon the work of John Mayne (2018; 2016), which has popularised these concepts within the evaluation field, and particularly as a tool to support the development of theories of change.

Within APRA, theories of change have been developed at the country and regional level using a Participatory Impact Pathways Analysis (PIPA) approach (Alvarez et al. 2010), which uses participatory approaches to develop a vision of change and identify potential pathways to impact placing particular emphasis on identifying influential stakeholders and strategies for engagement. The Diamond of Influence was developed to encourage researchers to reflect upon their engagement plans and think about how specific research messages responded to the opportunities and incentives in their national and regional policy contexts. This gave researchers an additional perspective to reflect on their progress against impact pathways, and provided a framework for ALRE to capture and share lessons learnt between country teams and generate insights to support country teams to prioritise and focus engagement activities moving into the final stages of APRA.

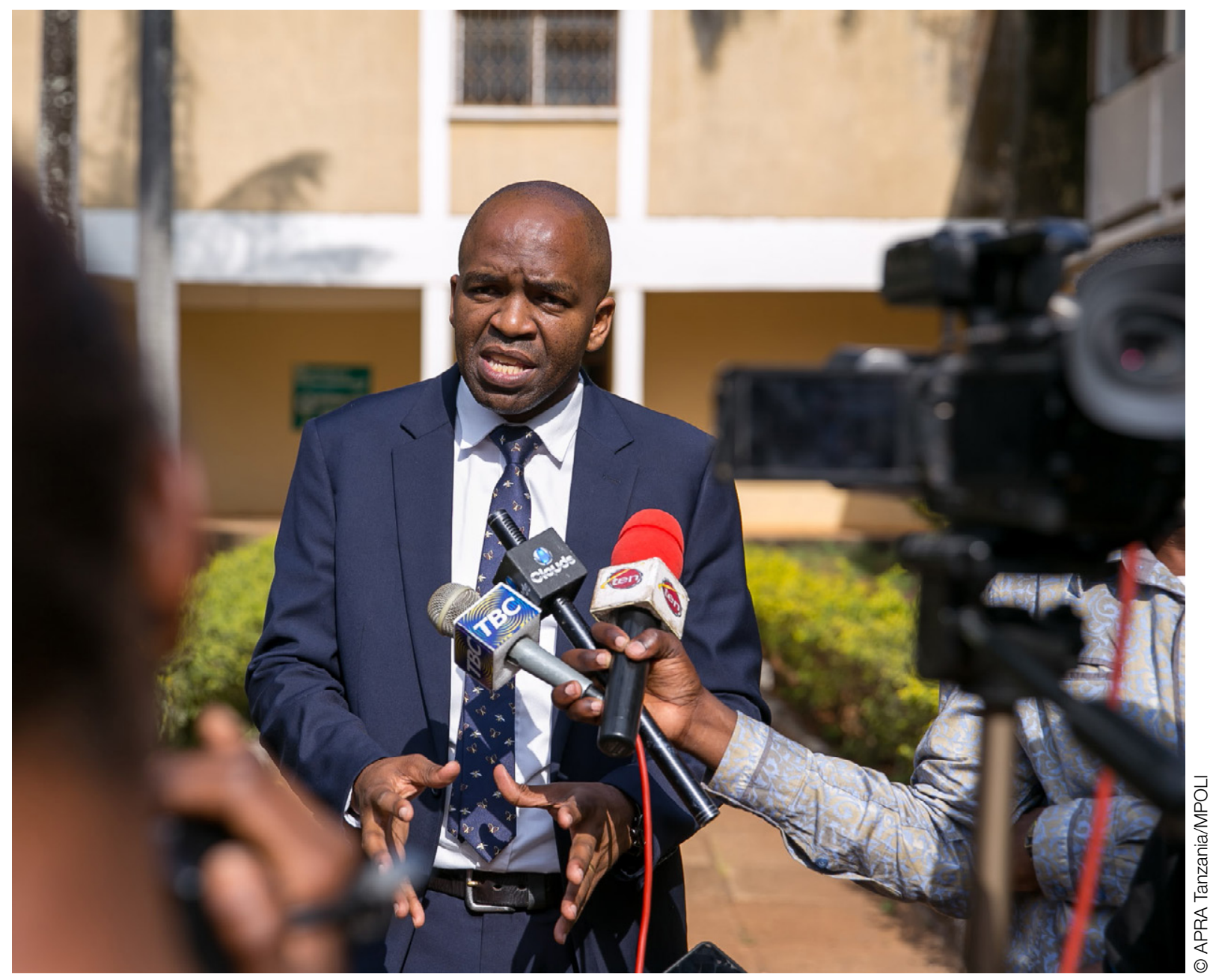




\section{The Diamond of Influence: a model for policy engagement}

The Diamond of Influence model emerged from consideration of how the COM-B concepts could be applied to deepen reflection around the specific questions that enhance relevance and effectiveness of APRA research. One of the key strengths of the COM-B model is that its four core elements are sufficiently broad that they have universal applications across fields and disciplines, including the research-policy interface. The Diamond of Influence also draws upon Georgalakis and Rose's (2019) framework which outlines three key qualities of research-policy partnerships in international development: adapting research evidence to policy priorities; collaborating towards a shared agenda; and sustained interaction and engagement to build trust.

Capabilities are linked to developing knowledge and skills. These two elements are at the core of the APRA programme, which works to deliver research that generates evidence and knowledge of the pathways to inclusive commercialisation in Sub-Saharan Africa (SSA), whilst supporting capacity and skills of partner researchers and research institutions. As such, in APRA, building capabilities - in terms of evidence and skills - is strongly linked to research activities and outputs. Professional and robust research that generates quality evidence provides a starting point for policy conversations and influencing but does not necessarily ensure either the relevance or effectiveness of this research from a policy perspective.

Opportunities are the external factors that support change. In the context of policy research, this can be interpreted as the framing of evidence in terms of how it contributes new insights to the broader policy context and responds to emerging issues that can create new opportunities to apply research evidence. Framing new evidence within existing policy conversations, or in response to emerging issues, is key to ensuring the policy relevance and effectiveness of research. This can be understood as policy adaptability that supports the use of research evidence in different policy spaces and for different audiences (Georgalakis and Rose 2019).

Motivation is about the internal cognitive processes that support decision-making and inform the lens through which decision-makers view research. It is therefore valuable to understand key stakeholders' incentives and the perspectives from which they will approach evidence. Identifying policy champions and finding mechanisms to increase their ownership or co-ownership of evidence and key findings and messages is therefore key to ensuring relevance and effectiveness. This aligns with Georgalakis

\section{Figure 1 The Diamond of Influence}

Applying COM-B to the research-policy interface

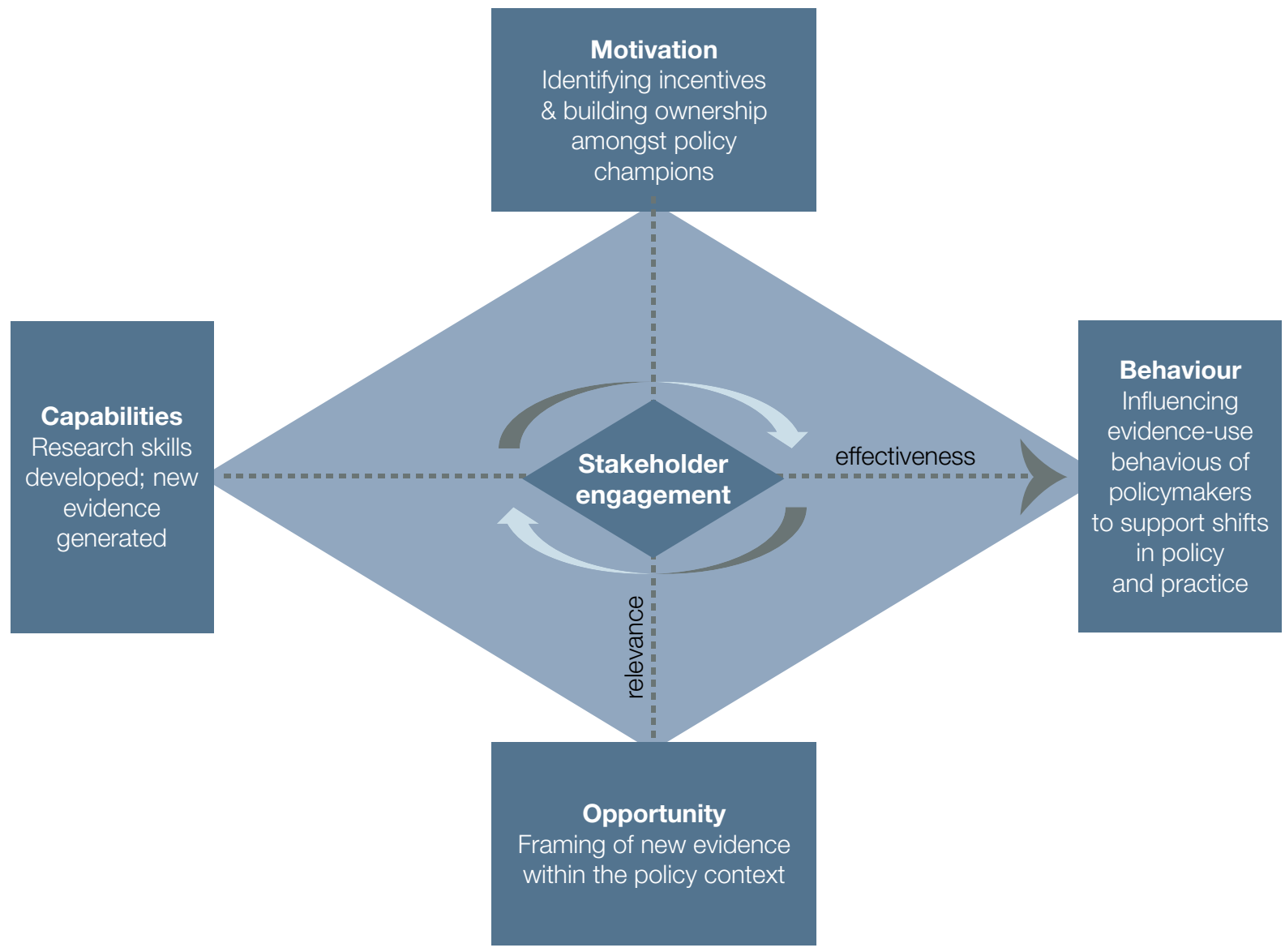




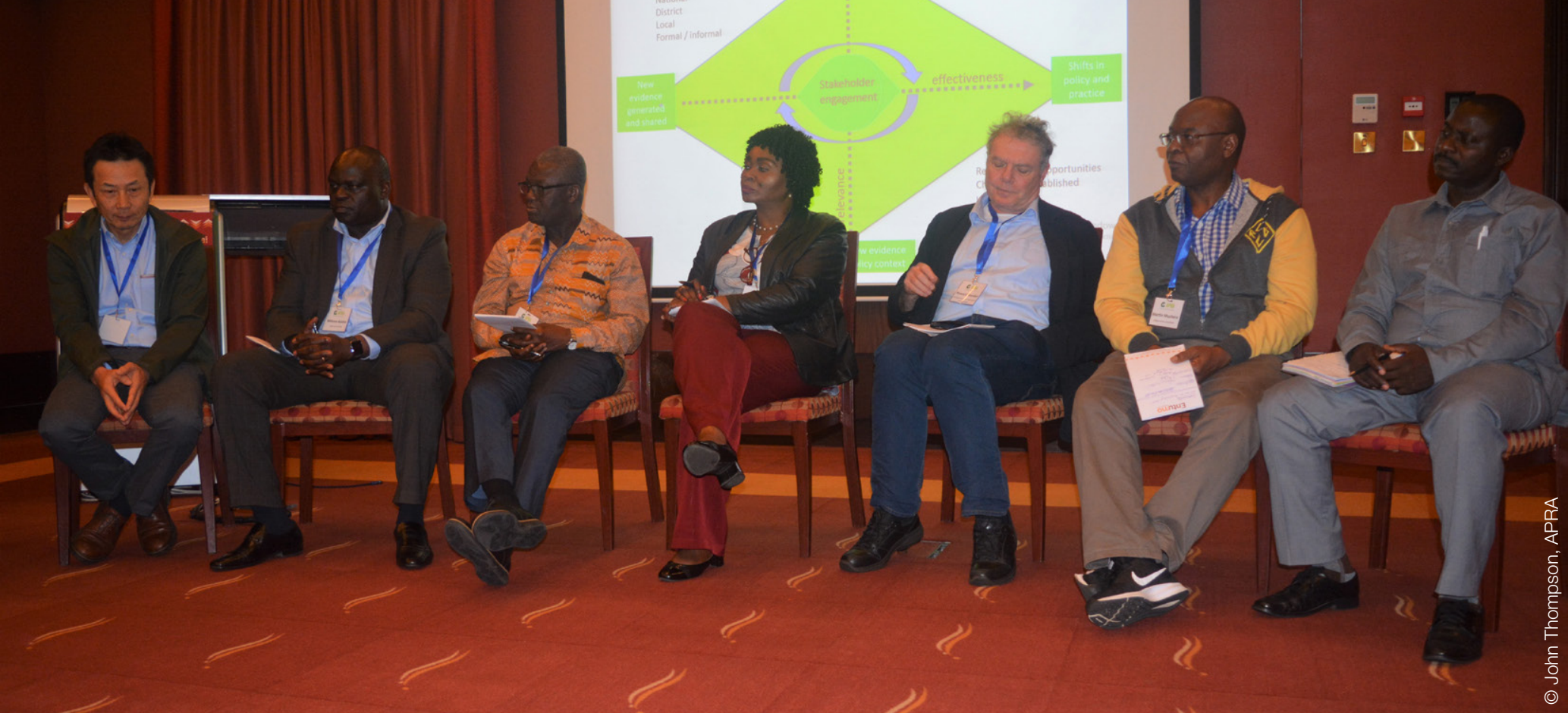

to Iramba and Mkalama districts in Singida region, including processor associations (CEZOSOPA TASUPA), business associations (FAIDAMALI), sunflower seed dealers and the Ministry of Agriculture at the national level. The APRA findings were framed by national policy decisions on incentives to produce edible oils, including import tariffs, seed development, and removal of VAT on processing. The research findings indicate that improving sunflower processing technology efficiency is necessary and the government should incentivise processors to upgrade their technology by exempting import duty on processing machinery. Greater emphasis is also needed on the use of improved seed varieties to ensure the supply of high yield sunflower varieties. At the local government level, improving warehouse and storage facilities should be a priority to ensure most farmers are selling sunflower off-season at a competitive price. APRA Tanzania is working with the advocacy group ANSAF (the Agricultural Non-State Actors Forum) to share their findings using simple briefs to capture key findings and policy messages that resonate at both national and district level and bring a unique perspective of how national policies are experienced at the local and household level.

In Ghana, decentralisation creates an opportunity for influence at the district level. Engaging at the national level is seen to be more challenging as the Ministry of Food and Agriculture does not send decision-makers to meetings. It is more feasible to access the Executive Director of the District Assembly, and create spaces at this level to discuss APRA findings of how farmers respond to market incentives, particularly as this finding challenges national perceptions. APRA research into oil palm highlighted how access to processing facilities increased the resilience of producers to price fluctuations. APRA research also highlighted the gender dynamics of oil palm processing with a strong role for women in artisanal processing but dominance of men linked to the use of machinery.
This issue of scaling can also apply to how national level policy solutions respond to the regional priorities of multilaterals. Establishing how research evidence provides new insights to existing or parallel research at national and regional levels may create potential avenues of influence that can demonstrate the relevance of key findings to inform decision-making and investments at different levels. Engaging with different policy priorities across different levels requires strong understanding of the dynamics and drivers at each level and agility to create connections that demonstrate how local experiences and insights contribute to national level conversations, as well as how regional priorities influence decisions at national levels and their impact on local level decision-making.

In Nigeria, national policy has prioritised diversification of the economy to reduce dependence on oil revenue, increasing the importance of cocoa production as an export crop. However cocoa production levels are down. APRA researchers are engaging at the national level with the Federal Ministry of Agriculture and Natural Resources to discuss the need to build resilient value chains in the context of the African Continental Free Trade Area Agreement. APRA researchers are also working at the community and local level in Nigeria, bringing together local-level associations with state-level actors in the value chain to understand the different drivers, challenges and behaviours of different stakeholders. Land tenure has been identified as key to promoting investment but farmers are unwilling to invest as they can be moved off their land after seven years and land security is being negatively affected by gold prospecting and extractivism. Bringing together stakeholders across different levels creates opportunities to build a shared vision of change and shared understanding of problems and first-hand consideration of potential solutions. For example, APRA researchers held meetings with regional police to resolve immediate 


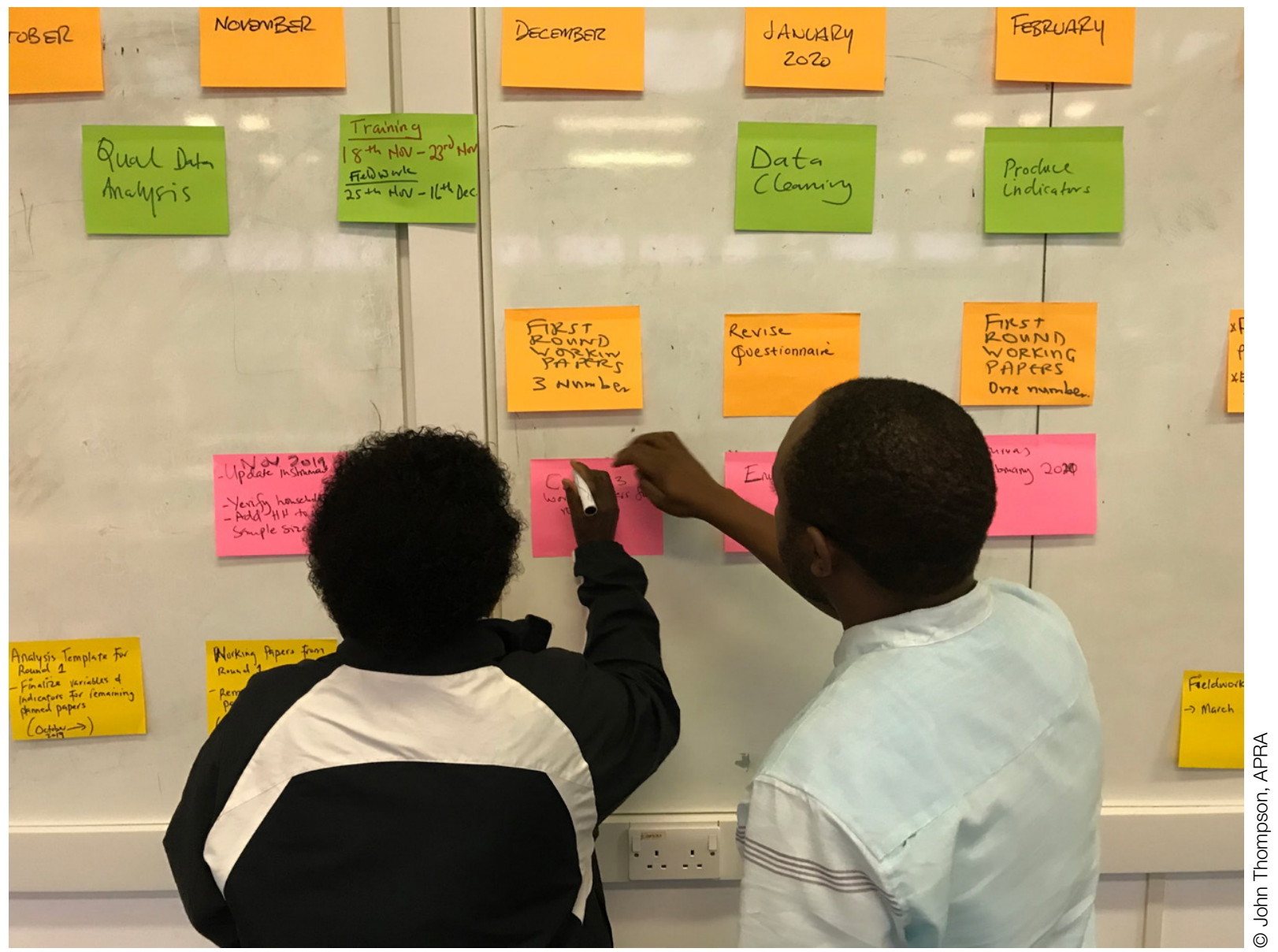

build their ownership of eventual results. This ongoing engagement builds policy audiences' awareness of research processes and provides them with an opportunity to advise and shape the focus and framing of research to respond to their specific needs and priorities, which can help overcome the challenge of closed ears if evidence challenges the existing status quo and vested interests of potential policy champions. Early engagement can help to bring champions on board, in order to anticipate and understand their incentives and disincentives to respond to emerging evidence and recommendations, and build relationships that have potential to increase their ownership and endorsement of evidence and promote emerging policy recommendations.

In Nigeria, one particular APRA team convened an Advisory Board that brings together influential civil servants and business leaders to provide guidance on how to translate APRA evidence into policy action. The Advisory Board meets bi-annually, supported by ongoing communication through a WhatsApp group, and the Board is tasked with assignments to identify mechanisms to translate APRA evidence on the potential role of medium-scale farms in enhancing the process of agricultural commercialisation and smallholder transformation into actionable recommendations. APRA's evidence suggests that, while the transition from small-scale to medium-scale farming has been occurring at a slow pace, encouraging growth of medium-scale farms provides an important pathway to increased agricultural commercialisation and thus policy should focus on accelerating the process. Increased labour and land productivity is found to be associated with increasing scale of operation; consequently, the growth of medium-scale farms could bring about improved livelihoods of farming households through increasing wages and returns to family labour. Findings also suggest that the growth of mediumscale farms could significantly impact smallholder input use decisions, output yields and sales prices through positive knowledge spill-over and coordination effects. The relationship between APRA researchers and the Advisory Board has opened up new opportunities and led to invitations to participate in other policy spaces.

\section{Bringing diverse voices together to build consensus}

Influencing policy conversations often involves bringing diverse voices together and identifying a common vision around a specific issue. If the key stakeholders are united around the value of a specific approach, then this creates a strong mandate to influence policy audiences. Mobilising and feeding research into multiple nodes of influence outside the state can amplify the voices and pressure to increase receptivity to policy recommendations amongst key decision-makers. Bringing together multiple constituents across the state, private sector, academia and civil society can create a strong advocacy movement but also requires domesticating ideas and language to different audiences.

In Malawi, APRA has worked with the Civil Society Agricultural Network, to identify activities and 
opportunities as they happen. This broad engagement resulted in media engagement and presentation of APRA evidence, which led to a request to share APRA evidence with the Strategic Agricultural Plan.

The APRA Malawi team also strengthened their relationship with media through a workshop with print and online journalists, which introduced journalists to a political-economy perspective to support them in communicating Malawi's experience of agricultural commercialisation to their readers in order to extend the reach of the research and systematically communicate findings to both farmers and policymakers. This increased visibility led to invitations for APRA Malawi to attend national policy dialogue forums.

APRA Malawi's participation at a State Agricultural Fair organised by the Food and Agriculture Organization of the United Nations (FAO) helped to raise awareness of the commercialisation challenges faced by farmers' organisations and the need to help re-strategise to gain access to markets.

In Zimbabwe, APRA researchers recognised the need to engage with different audiences with different messages. In particular, this work emphasised the need to engage with banks through the Bankers Association to address their reluctance to extend credit. In addition, the changing forms of farm labour relations and the diverse ways in which farmers eke out a livelihood present new labour supply and wage demand challenges. Engaging with farmers' organisations and farmworker associations with targeted messages was seen as crucial in this regard. The Zimbabwe team

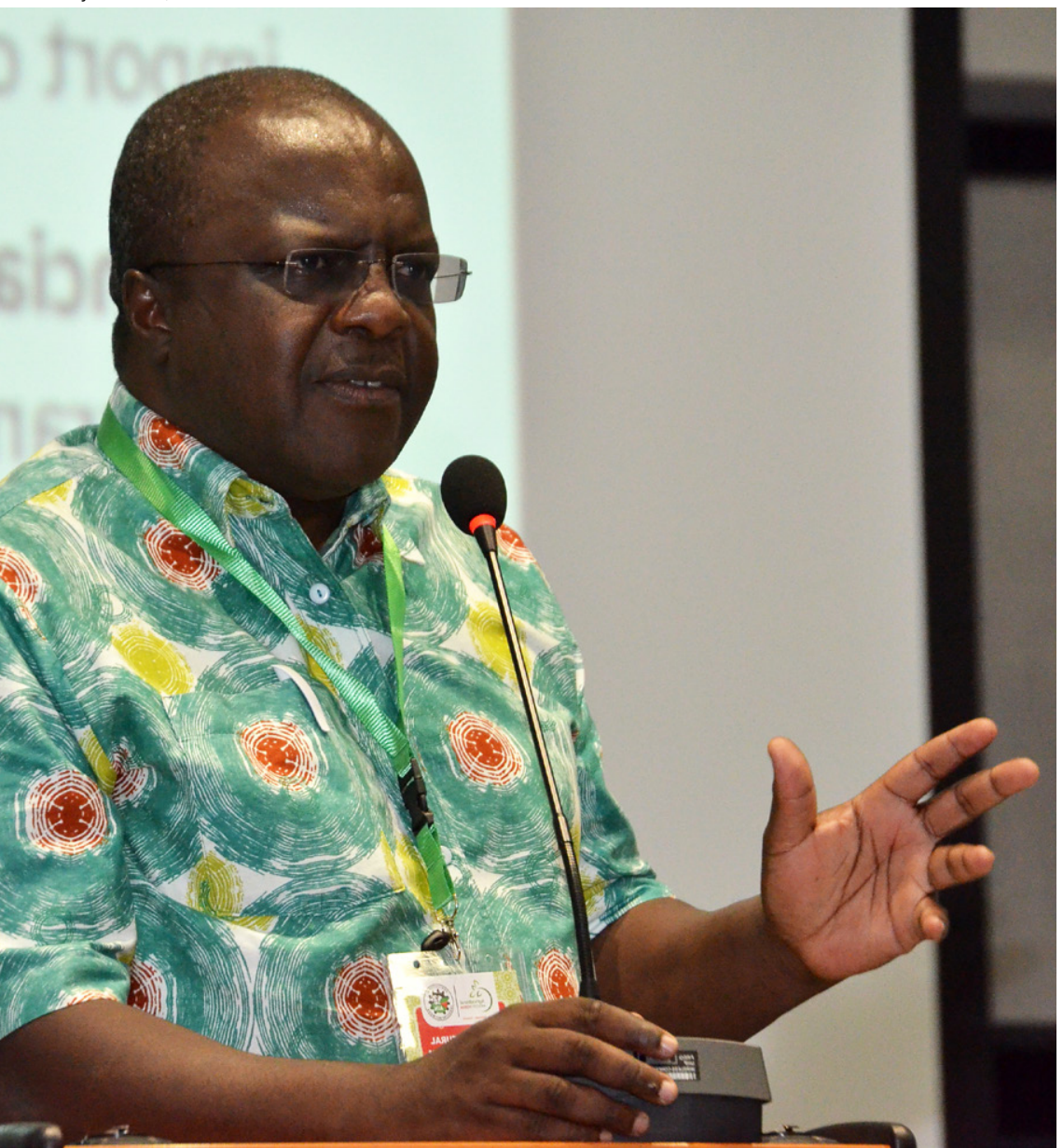

also used a participatory approach to work on climate change, which resulted in much stronger engagement of farmers in the research process and stronger learning and evaluation of the processes. Agricultural extension officers who attended focus group discussions appreciated emerging climate vulnerability and resilience dilemmas, prompting the need to develop new messages for government policymaking audiences. Broader stakeholder participation led to stronger ownership amongst farmers' groups, which was beneficial in crafting key messages for policymakers. APRA researchers are engaging with national policy framework alongside key stakeholders in government and FAO.

\section{Building momentum around research evidence}

An additional dimension to incentivise the use and uptake of research findings or methods is to build momentum through horizontal sharing of evidence of peers in academia or research. Whilst many academic conferences focus on sharing specialist knowledge and technical expertise, which can have limited interest to policymakers, there are also instances in which this exchange and uptake by peers in academia and research can act as a validation of a new idea or approach. Building momentum around ideas through collaborating with other research institutes can elevate the profile of new evidence and generate broader interest that has stronger potential to capture policymakers' attention and interest.

APRA research has been adopted and adapted by EIAR, which used APRA survey tools on rice commercialisation in Ethiopia. APRA is also working in collaboration with other research institutes including the International Rice Research Institute, the Coalition for African Rice Development, and the Japan International Cooperation Agency-funded EthioRice project to plan a Regional Rice Conference. Due to COVID-19, this has been postponed to 2021. However, this collaboration has already generated strong donor and policymaker interest and will create an opportunity to highlight the key policy messages, explore synergies and develop new ideas as well as collectively explore practical solutions emerging from across these research programmes.

\section{Stakeholder engagement to increase relevance and effectiveness of APRA research}

In reality, there is very much a symbiotic relationship between understanding the motivation and incentives of policymakers and framing research in a way that responds to their priorities and building ownership. The common thread which links these approaches to policy influencing is stakeholder engagement, which connects all of the different strategies to identify opportunities and create motivation in order to increase the relevance of 
Figure 2 Stakeholder engagement drives opportunities and motivation

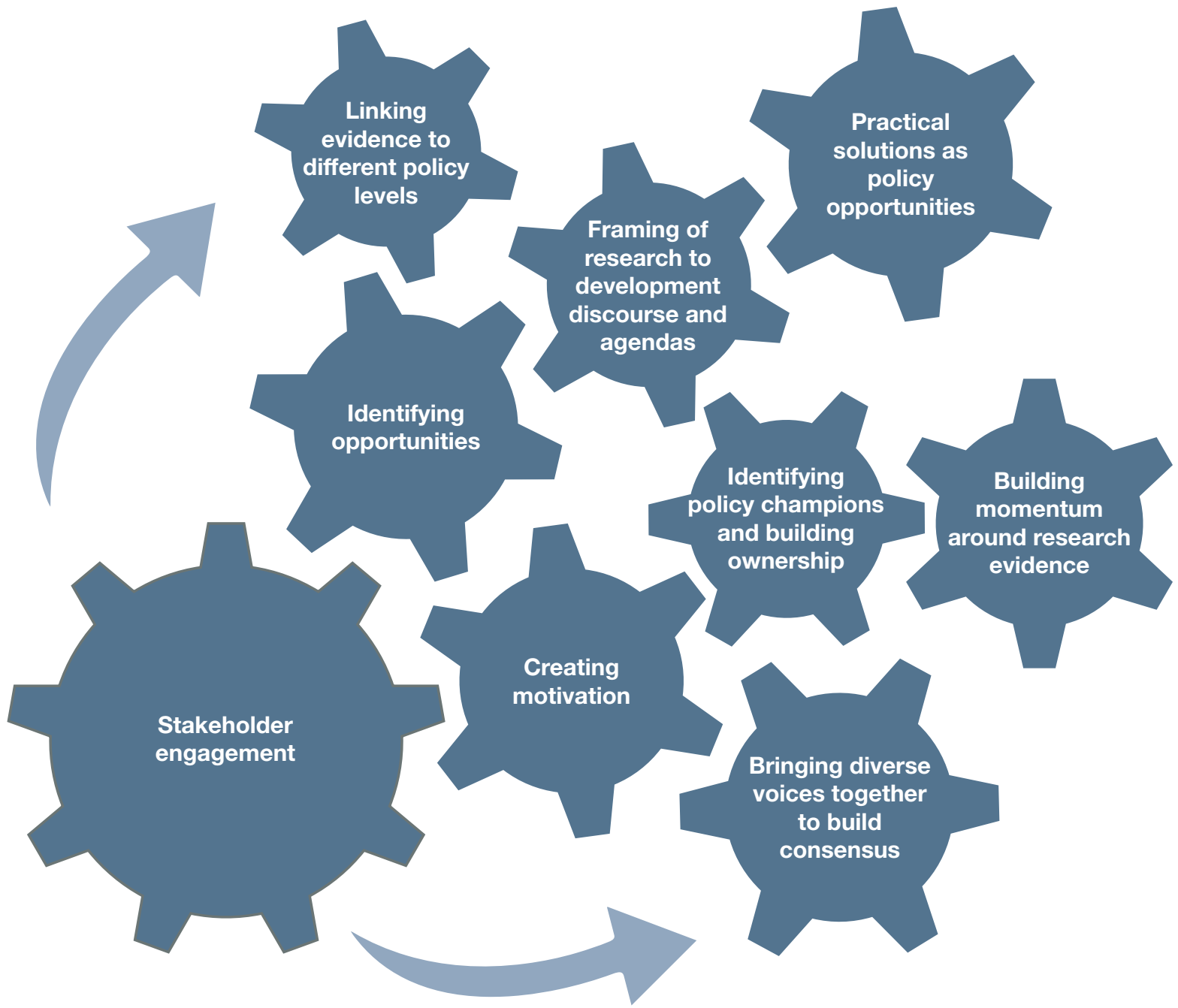

Source: Author's own

research outlined above as illustrated in Figure 2. These strategies are mutually reinforcing and can support researchers to think beyond the research process and place greater emphasis on the use and uptake of the evidence generated to support policy outcomes.

Stakeholder engagement requires investment in time and resources, as well as developing strong facilitation and communication skills to capture and maintain the attention of different actors with a stake in research findings. Research programmes that are successful in building skills and generating new knowledge can create capabilities, but the impacts will be limited unless this evidence is supported by a strong engagement strategy that seeks to build lasting relationships and bring different voices to the table. Understanding the motivation and incentives of different stakeholder groups and identifying potential policy champions, as well as framing emerging findings in the context of existing and emerging policy opportunities, are critical with regards to the relevance of research findings to respond to policy priorities and questions. Investing energy and resources in achieving this is essential to ensure the most effective use of research evidence to change stakeholders' perspectives and behaviours towards a shift in policy and programmatic discourse and delivery.
APRA researchers are applying these different strategies and approaches as they build relationships and refine their research messages to resonate with policy opportunities at different levels of government. Continuous reflection and iteration of impact pathways, particularly in response to COVID-19, places strong emphasis on adjusting research plans, key messages and engagement strategies to current country realities to ensure ongoing relevance of APRA evidence and communications approaches. Prioritising relevance will provide strong sign posts to support future ALRE work to evaluate the effectiveness of APRA research to influence policies to promote inclusive agricultural commercialisation in Africa. 


\section{References}

Alvarez, S.; Thiele, G., Mackay, R. and Douthwaite, B. (2010) 'Participatory Impact Pathways Analysis: A Practical Method for Project Planning and Evaluation', Development in Practice 20(8): 946-958

Clark, L. and Goodier, S. (2019) The Delta of Impact: Mapping a Meta-Impact Pathway to Uncover Six Stepping Stones of Research Impact, Working Paper: Expert Analysis from the Impact Initiative, Brighton: IDS and The Impact Initiative

Georgalakis, J. and Rose, P. (2019) 'Identifying the Qualities of Research-Policy Partnerships in International Development - A New Analytical Framework', in J. Georgalakis and P. Rose (eds.), Exploring Research-Policy Partnerships in International Development, IDS Bulletin 50(1): 1-19, https://doi.org/10.19088/1968-2019.103

Mayne, J. (2016) The Capabilities, Opportunities and Motivation Behaviour-Based Theory of Change Model, https://www.researchgate.net/publication/301701597_The_Capabilities_Opportunities_and_Motivation_ Behaviour-Based_Theory_of_Change_Model (accessed 1 October 2020)

Mayne, J. (2018) The COM.B ToC Model4, https://www.researchgate.net/publication/323868561_The_COMB_ ToC_Model4 (accessed 1 October 2020)

Michie, S.; van Stralen, M.M. and West, R. (2011) 'The Behaviour Change Wheel: A New Method for Characterising and Designing Behaviour Change Interventions, Implementation Science 6, 42, https://doi. org/10.1186/1748-5908-6-42

OECD. (1991) DAC Principles for Evaluation of Development Assistance, Paris: Organisation for Economic Cooperation and Development, https://www.oecd.org/dac/evaluation/2755284.pdf (accessed 1 October 2020)

OECD/DAC. (2019) Better Criteria for Better Evaluation Revised Evaluation Criteria Definitions and Principles for Use, Paris: Organisation for Economic Co-operation and Development Development Assistance Committee, https://www.oecd.org/dac/evaluation/revised-evaluation-criteria-dec-2019.pdf (accessed 1 October 2020)

Patton, M.Q. (2010) Developmental Evaluation. Applying Complexity Concepts to Enhance Innovation and Use, New York: Guilford Press 
Clark, L. (2020) The Diamond of Influence: A Model for Exploring Behaviour in Research to Policy Linkages, ALRE Research Note 1, Brighton: Future Agricultures Consortium

(c) APRA 2020

ISBN: 978-1-78118-711-1

DOI: $10.19088 / A P R A .2020 .011$

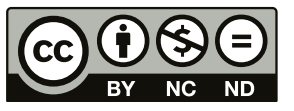

This is an Open Access report distributed under the terms of the Creative Commons Attribution Non Commercial No Derivatives 4.0 International licence (CC BY-NC-ND), which permits use and distribution in any medium, provided the original authors and source are credited, the work is not used for commercial purposes, and no modifications or adaptations are made. https://creativecommons.org/licenses/by-nc-nd/4.0/legalcode

If you use the work, we ask that you reference the APRA website (www.future-agricultures.org/apra/) and send a copy of the work or a link to its use online to the following address for our archive: APRA, Future Agricultures Consortium, University of Sussex, Brighton BN1 9RE, UK (apra@ids.ac.uk).

Agricultural Policy Research in Africa (APRA) is a programme of the Future Agricultures Consortium (FAC) which is generating new evidence and policy-relevant insights on more inclusive pathways to agricultural commercialisation in Sub-Saharan Africa. APRA is funded with UK aid from the UK Foreign, Commonwealth \& Development Office (FCDO) and will run from 2016-2022.

The APRA Directorate is based at the Institute of Development Studies (IDS), UK (www.ids.ac.uk), with regional hubs at the Centre for African Bio-Entrepreneurship (CABE), Kenya, the Institute for Poverty, Land and Agrarian Studies (PLAAS), South Africa, and the University of Ghana, Legon. It builds on more than a decade of research and policy engagement work by the Future Agricultures Consortium (www.future-agricultures.org) and involves more than 100 researchers and communications professionals in Africa, UK, Sweden and USA
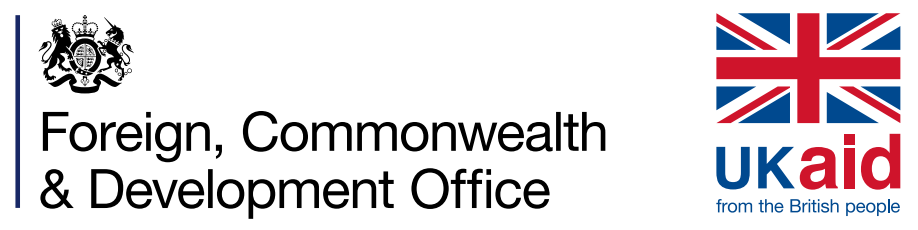